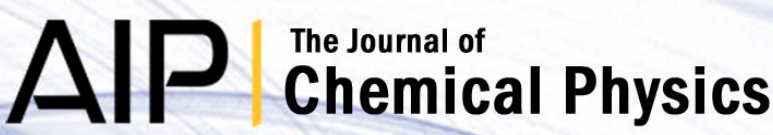

\section{Evidence for several dipolar quasi-invariants in liquid crystals}

C. J. Bonin, C. E. González, H. H. Segnorile, and R. C. Zamar

Citation: J. Chem. Phys. 139, 144907 (2013); doi: 10.1063/1.4823994

View online: http://dx.doi.org/10.1063/1.4823994

View Table of Contents: http://jcp.aip.org/resource/1/JCPSA6/v139/i14

Published by the AIP Publishing LLC.

Additional information on J. Chem. Phys.

Journal Homepage: http://jcp.aip.org/

Journal Information: http://jcp.aip.org/about/about_the_journal

Top downloads: http://jcp.aip.org/features/most_downloaded

Information for Authors: http://jcp.aip.org/authors
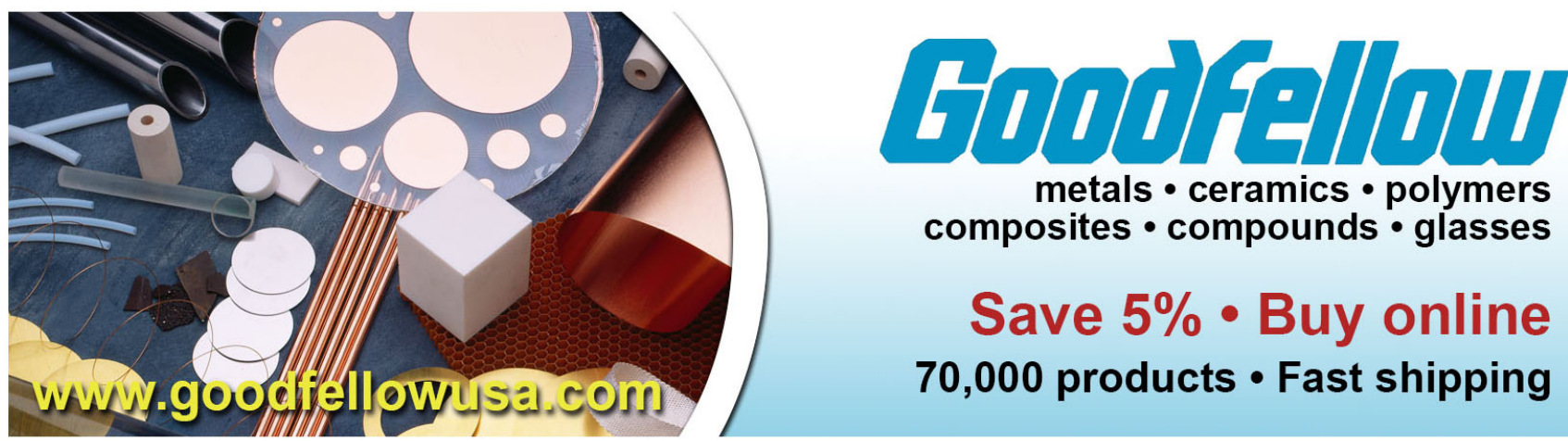

metals • ceramics - polymers composites • compounds • glasses

Save $5 \%$ • Buy online 70,000 products $\cdot$ Fast shipping 


\title{
Evidence for several dipolar quasi-invariants in liquid crystals
}

\author{
C. J. Bonin, ${ }^{1,2}$ C. E. González, ${ }^{1,3}$ H. H. Segnorile, ${ }^{1,3}$ and R. C. Zamar ${ }^{1,3, a)}$ \\ ${ }^{1}$ Facultad de Matemática, Astronomía y Física, Universidad Nacional de Córdoba (FaMAF) M.Allende y H. de \\ la Torre, Ciudad Universitaria, X5016LAE Córdoba, Argentina \\ ${ }^{2}$ Instituto de Desarrollo Tecnológico para la Industria Química, CONICET, Santa Fe, Argentina \\ ${ }^{3}$ Instituto de Física Enrique Gaviola, CONICET, Córdoba, Argentina
}

(Received 10 June 2013; accepted 18 September 2013; published online 11 October 2013)

\begin{abstract}
The quasi-equilibrium states of an observed quantum system involve as many constants of motion as the dimension of the operator basis which spans the blocks of all the degenerate eigenvalues of the Hamiltonian that drives the system dynamics, however, the possibility of observing such quasiinvariants in solid-like spin systems in Nuclear Magnetic Resonance (NMR) is not a strictly exact prediction. The aim of this work is to provide experimental evidence of several quasi-invariants, in the proton NMR of small spin clusters, like nematic liquid crystal molecules, in which the use of thermodynamic arguments is not justified. We explore the spin states prepared with the Jeener-Broekaert pulse sequence by analyzing the time-domain signals yielded by this sequence as a function of the preparation times, in a variety of dipolar networks, solids, and liquid crystals. We observe that the signals can be explained with two dipolar quasi-invariants only within a range of short preparation times, however at longer times liquid crystal signals show an echo-like behaviour whose description requires assuming more quasi-invariants. We study the multiple quantum coherence content of such signals on a basis orthogonal to the z-basis and see that such states involve a significant number of correlated spins. Therefore, we show that the NMR signals within the whole preparation timescale can only be reconstructed by assuming the occurrence of multiple quasi-invariants which we experimentally isolate. (C) 2013 AIP Publishing LLC. [http://dx.doi.org/10.1063/1.4823994]
\end{abstract}

\section{INTRODUCTION}

Quasi-equilibrium states (QE) found in Nuclear Magnetic Resonance (NMR) of strongly interacting nuclear spin systems are quantum states which do not evolve under the system Hamiltonian, thus they can be represented by a reduced spin density operator diagonal in blocks in the eigenbasis of the spin-environment Hamiltonian. ${ }^{1-3}$ These states only evolve due to spin-lattice relaxation, over a time scale much longer than the one needed for the build-up of QE. Since the early times of the spin thermodynamics theory, QE states have been widely used in solid state NMR especially when the dipolar interactions play an important role. ${ }^{4,5}$ In particular, the many-spin character of dipolar order has attracted recent attention, e.g., generation of second-order dipolar order in three-spins systems, ${ }^{6,7}$ investigation of the many-spin dynamics of nuclear spins in solid state multiple quantum coherence (MQC) NMR, ${ }^{8}$ and computational modelling of the dynamics of large networks of strongly coupled spins. ${ }^{9}$ In this work we address the study of QE in nematic liquid crystals.

One of the experimental NMR procedures to prepare and detect dipolar QE states in NMR is the Jeener-Broekaert pulse sequence $(\mathrm{JB}){ }^{4}$ Briefly, this technique consists of the phase-shifted radiofrequency (rf) pulses: $90_{x}-\tau-45_{y}$ $-t_{e}-45_{y}-t$. The first pulse creates single-spin singlequantum coherences in the spin system state (or "uncorrelated" coherences ${ }^{10}$ ), which evolve in the rotating frame mainly under the dipole spin-spin Hamiltonian during $\tau$.

$\overline{{ }^{a)} \text { Electronic address: zamar@famaf.unc.edu.ar }}$
Along this period, multi-spin single-quantum coherences can develop, and the second $45_{y}$ pulse transforms part of the coherences just created into multi-spin order., ${ }^{2,10,11}$ Two important processes occur during the evolution period $t_{e}$, along different timescales: decoherence and relaxation. The third pulse converts QE states into observable single quantum coherence. Quantum decoherence is the microscopic phenomenon which brings the spin density matrix to a QE form over an early timescale after the second pulse. Spin lattice relaxation processes finally draw the spin system towards thermodynamic equilibrium.

It is an experimental fact that for short preparation times $\tau \ll 1 / \omega_{D}$ in the JB sequence, the dipolar signal observed after the third pulse is proportional to the time derivative of the Free Induction Decay signal (FID) ${ }^{4}$ which indicates that the prepared state is very similar to the secular (high field) dipolar energy. In a variety of samples this is the only observable dipolar QE state. Two dipolar QE states, were instead observed both on hydrated salts, ${ }^{5,12}$ and on liquid crystals (LCs). ${ }^{13,14}$ The occurrence of "intra-pair" and "inter-pair" QE states in hydrated salts was associated with the distribution of the proton pairs of the water molecules in the lattice, conforming a system of weakly interacting spin pairs. The spin system of LCs instead, comprises the few proton spins within each molecule, which display a hierarchy of dipolar couplings. This kind of dipolar network is different from hydrated salts and a model of spin pairs does not strictly proceed, however, the dipolar interactions in the spin cluster can be classified approximately into strong and weak interactions. In fact, the short time spin dynamics was consistently 
described by assuming that the dipolar Hamiltonian can be partitioned into two mutually commuting and orthogonal parts, the "strong" $\mathcal{H}_{\mathcal{S}}$ and the "weak" $\mathcal{H}_{\mathcal{W}}$ terms. ${ }^{15}$ Since these operators can be chosen so that they commute with the total spin Hamiltonian, they can be considered as constants of the motion, excluding relaxation effects. For this reason, they are called quasi-invariants (QIs). ${ }^{5,16}$

In early works on dipolar order relaxation in LCs, the experiment was interpreted in terms of a single QI, the dipolar energy of an ensemble of isolated representative strongly coupled spin pairs, $\mathcal{S} ;{ }^{17}$ however, the observed dependence of the relaxation times on $\tau$ could not be explained within this view. ${ }^{18}$ Later works showed that other state $\mathcal{W}$ prepared by setting the preparation time to the one at which the $\mathcal{S}$ order vanishes, also behaves as a QI. In fact, it relaxes exponentially towards equilibrium, with a characteristic time $T_{1 \mathcal{W}}$ which is also of the order of $T_{1 D} \cdot{ }^{14}$ Besides, experiments where the MQCs of the states prepared with the JB sequence are encoded in orthogonal, $X$-basis, ${ }^{10}$ carried out in nematic $5 \mathrm{CB},{ }^{19}$ confirmed that the tensor structure of the $\mathcal{S}$ state corresponds to two-spin dipolar order while the $\mathcal{W}$ state shows higher order quantum coherences, all of which relax towards equilibrium with the same decay constant $T_{1 \mathcal{W}}$. This indicates that for short preparation times the two-spin order prevails while higher order spin correlations are growing. This second QI, produces discernible signals of multi-spin order, created within a time window $\tau$ where the dipolar order vanishes, similar to the hydrated crystals studied in Refs. 5 and 12.

All these results support the assumption that the density operator of nematic LCs can be written, for times $t_{e}$ greater than the decoherence time scale, in the form ${ }^{15}$

$$
\rho_{D} \propto \mathbf{1}-\beta_{Z} \mathcal{H}_{Z}-\beta_{\mathcal{S}}(\tau) \mathcal{H}_{\mathcal{S}}-\beta_{\mathcal{W}}(\tau) \mathcal{H}_{\mathcal{W}} .
$$

Although the analytic form of operators $\mathcal{H}_{\mathcal{S}}$ and $\mathcal{H}_{\mathcal{W}}$ is not known, the experimental behaviour of the dipolar signal of 5CB was shown to be compatible with Eq. (1). However, the agreement is only valid for preparation times $\tau \leq 80 \mu$ s. $^{14,15}$ The same kind of partial agreement is also present in a variety of nematic LCs, as shown in Sec. III.

In principle, in a cluster of $N$ dipole interacting spins $1 / 2$, as the protons of a typical LC molecule, there are about $2^{N}$ quantum constants of motion, the exact number depends on the degeneracy of the dipolar Hamiltonian. ${ }^{2}$ That is, such a number of spin operators are needed to span the commutative space of the dipolar Hamiltonian. The experimental detection of two dipolar QIs, is consistent with this idea but accordingly, it should also be possible to prepare new QE states from the initial Zeeman order, which should be observable for longer preparation times in the JB experiment, as long as the JB signal is detectable.

The aim of this work is to explore the spin states prepared for long preparation times in the JB experiment, looking for experimental evidence of multiple QIs that can be expected for dipole-coupled spin clusters. The analysis is based on an exhaustive study of the time-domain dipolar signals in a variety of dipolar networks and on the analysis of the MQC content of the QE states prepared with the JB sequence.

Providing insight on the nature of the QIs of a spin cluster can be useful both for applications as for basic re- search. QE states are relevant observables of the spin system, providing relaxation parameters useful to study molecular motion in LC mesophases through their dependence on temperature and magnetic field. ${ }^{14,20,21}$ They have been proposed as initial states for the excitation of MQC in NMR experiments ${ }^{8,22,23}$ and used in spin counting experiments. ${ }^{10,19}$ Besides, these states have been proposed as alternative to the Zeeman order in magnetic resonance imaging. ${ }^{24}$ Implementation of noiseless quantum memories and multi-spin quantum register relies on the possibility of manipulating multispin correlated entities which are unperturbed by decoherence processes. $^{25-28}$ From a basic viewpoint, the physics of systems with few degrees of freedom coupled to a quantum environment attracts today's attention of a widespread community because of the potentiality for applications such as quantum devices and quantum information processing ${ }^{25,29,30}$ and, significantly, also because these systems are testbeds for studying fundamental aspects as irreversibility, equilibration, and thermalization. $1,31,32$

Section II contains the definition of the QI operators and their relation with the NMR signals in the JB experiment. In Sec. III we present an experimental survey of the manifestation of multiple QIs and propose a method to isolate them in samples with different spin network geometry. The nature of the different QIs is examined by means of relaxation experiments and by studying the MQC content on the $\mathrm{x}$-basis. In the Appendix we show that the signal after the JB sequence is symmetric in the preparation and observation times.

\section{QUASI-INVARIANTS}

The very nature of the QE states in LCs is nowadays an open issue and the manner in which these states are reached matters since it defines part of their properties. It was recently shown that quantum decoherence can provide an efficient mechanism by which the spin system attains a diagonal state in the basis of the spin-environment Hamiltonian, over a time scale which is intermediate between those governed by its own interactions, and thermalization ruled by thermal fluctuations of the environment. ${ }^{1,3}$ Relaxation brings the system to thermal equilibrium with the whole surrounding world over a much longer time scale, $t_{e} \gg \tau$, through energy exchange between the spins and the lattice. The characteristic lifetimes of dipolar QE states (dipolar order relaxation times) are comparable to the common Zeeman relaxation time.

The spin Hamiltonian of resonant nuclei in ordered systems such as solids and LC has an important contribution from the dipole-dipole coupling energy besides the Zeeman term,

$$
\mathcal{H}_{S}=\mathcal{H}_{Z}+\mathcal{H}_{D}
$$

where the Zeeman energy in units of $\hbar$ is $\mathcal{H}_{Z}=-\omega_{o} \mathbf{I}_{z}$, with $\omega_{o}$ the Larmor frequency. In the rotating frame description, ${ }^{33}$ the time evolution of any spin state is mainly driven by the dipolar Hamiltonian and often only by its secular part (high field approximation),

$$
\mathcal{H}_{D}^{0}=\sqrt{6} \sum_{i<j} D_{i j} \mathbf{T}_{20}^{i j}
$$


where $\mathbf{T}_{20}^{i j}$ is the zero component of a normalized irreducible spherical tensor of rank two, which in terms of the spin angular momentum operators is ${ }^{34,35}$

$$
\mathbf{T}_{20}^{i j}=\frac{1}{\sqrt{6}}\left[2 I_{z}^{i} I_{z}^{j}-\frac{1}{2}\left(I_{+}^{i} I_{-}^{j}+I_{-}^{i} I_{+}^{j}\right)\right] .
$$

In LCs the secular part of the dipolar coupling between nuclei $i$ and $j$ is

$$
D_{i j} \equiv\left\langle\frac{\mu_{o} \gamma^{2} \hbar}{4 \pi}\left(\frac{1-3 \cos ^{2} \theta_{i j}}{2 r_{i j}^{3}}\right)\right\rangle,
$$

with $r_{i j}$ the distance between spins, $\theta_{i j}$ the angle between the internuclear vector and the polarizing external magnetic field, and the angle brackets stand for an average over internal and reorientational molecular motions. Because of the rapid molecular motions the residual dipolar coupling in LC only involves protons within a molecule, then, the sum of Eq. (3) runs over protons within a molecule. Then, contrarily to solids, the spin system has few degrees of freedom, and the normal arguments used to support the $\mathrm{QE}$, based on equilibrium thermodynamics of a closed system do not apply to LC. ${ }^{17}$ Alternatively, the proton spins can be considered as an open quantum system, coupled to a large quantum molecular environment. Within this framework, the environment induced decoherence causes the irreversible decay of the spin density matrix to a diagonal-in-blocks form, over a timescale $t_{q e}$ intermediate between the quantum interference and relaxation. This hypothesis was theoretically formulated and experimentally confirmed recently on liquid crystal NMR,,${ }^{1,3,42}$ and is also consistent with the results of similar approaches from other areas. ${ }^{31,43}$

An arbitrary initial state $\rho_{0}$ of a closed system evolves, according to the Liouville equation, like

$$
\rho(t)=e^{-i \mathcal{H}_{D}^{0} t} \rho_{0} e^{i \mathcal{H}_{D}^{0} t} .
$$

Considering instead the spin system as an open quantum system implies that there is an observed system associated with a Hamiltonian $\mathcal{H}_{S}$ coupled to a larger reservoir with a Hamiltonian $\mathcal{H}_{L}$. The Hamiltonian of the whole system is

$$
\mathcal{H}=\mathcal{H}_{S}+\mathcal{H}_{S L}+\mathcal{H}_{L}
$$

where $\mathcal{H}_{S L}$ is the full quantum dipolar Hamiltonian with both the spin and lattice variables represented by quantum operators. ${ }^{1,31,42,43}$

Irreversible decoherence originates in the interaction of a finite quantum system with the environment within the essentially adiabatic regime ${ }^{1}$ (or "quasi-isolated" regime ${ }^{31}$ ), characterized by the commutation relations

$$
\left[\mathcal{H}_{S}, \mathcal{H}_{S L}\right]=0 ; \quad\left[\mathcal{H}_{L}, \mathcal{H}_{S L}\right] \neq 0,
$$

which can be assumed within a timescale $t_{q e}$ where $\left\|\left[\mathcal{H}_{S}, \mathcal{H}_{S L}\right]\right\| t_{q e} \ll 1$. This decoherence process attenuates the off-diagonal elements of the spin state (in the basis of the spin-lattice interaction Hamiltonian) while preserves the diagonal in blocks, leaving a density operator $\rho_{q e}$ which commutes with the spin Hamiltonian and is therefore a constant of motion. Since this state can only evolve within a longer timescale due to relaxation it is called a $\mathrm{QE}$ state. Owing to this property $\rho_{q e}$ can be formally expanded in an arbitrary basis which spans the commutative space of $\mathcal{H}_{D}^{0}$,

$$
\rho_{q e}(\tau)=\frac{1}{\mathcal{N}}\left(\mathbf{1}-\sum_{k} \beta_{k}(\tau) \mathcal{Q}_{k}\right),
$$

where $\mathcal{Q}_{k}$ are zero-trace operators which commute with the spin Hamiltonian in the rotating frame, $\left[\mathcal{Q}_{i}, \mathcal{H}_{D}^{0}\right]=0$ and we therefore call them QIs. They satisfy the orthogonality relations

$$
\operatorname{Tr}\left\{\mathcal{Q}_{k} \mathcal{Q}_{k^{\prime}}\right\}=\delta_{k, k^{\prime}} \operatorname{Tr}\left\{\mathcal{Q}_{k}^{\dagger} \mathcal{Q}_{k}\right\},
$$

the coefficients $\beta_{k}(\tau)$ are

$$
\beta_{k}(\tau)=-\mathcal{N} \operatorname{Tr}\left\{\mathcal{Q}_{k}^{\dagger} \rho_{q e}(\tau, t)\right\} / \operatorname{Tr}\left\{\mathcal{Q}_{k}^{\dagger} \mathcal{Q}_{k}\right\},
$$

and $\mathcal{N}=\operatorname{Tr}\{\mathbf{1}\}$ is the normalization factor.

From an experimental viewpoint, such a state of "internal" QE in the JB experiment is reached when a time $t_{e}$ after the second pulse longer than decoherence has elapsed.

It is important to remark that in spite of the mathematical similitude, Eq. (4) is not intended to be the first order term of a high temperature series expansion of the thermodynamical Gibbs distribution. It is worth mentioning that the QE density operator of Eq. (4) is also formally similar to the one proposed within the spin-thermodynamics approach, where it is also claimed that "there may be relevant quantities other than the total energy that are constants of the motion." ${ }^{41}$ However, the hypotheses which support the occurrence of QE in solids via spin-thermodynamics are not satisfied in the case of LC because of the small number of degrees of freedom of the spin system.

The NMR signal detected in phase with the read pulse, compatible with Eq. (4) is

$$
\begin{aligned}
S(t) & =\left\langle I_{y}(t)\right\rangle=\operatorname{Tr}\left\{U(t) P_{r} \rho_{q e} P_{r}^{\dagger} U(t)^{\dagger} I_{y}\right\} \\
& =\sum_{i} \beta_{i}(\tau) \operatorname{Tr}\left\{U(t) P_{r} \mathcal{Q}_{i} P_{r}^{\dagger} U^{\dagger}(t) I_{y}\right\} / \mathcal{N} \\
& =\sum_{i} \beta_{i}(\tau) \mathcal{F}_{i}(t) / \mathcal{N},
\end{aligned}
$$

where $P_{r}$ represents the read pulse and $U(t)=\exp \left\{-i \mathcal{H}_{D}^{0} t\right\}$ is the evolution operator during the observation period. Within this view, coefficients $\beta_{i}(\tau)$ can be interpreted as the weight with which operator $\mathcal{Q}_{i}$ contributes to the overall NMR signal for a given preparation time, and $\mathcal{F}_{i}(t)$ as the contribution of the $i$ th QI to the dipolar signal. Although the sum in the second line of Eq. (5) formally runs over all the commutative space of $\mathcal{H}_{D}^{0}$, the actual number of QIs needed for describing $S(t)$ cannot be predicted a priori and also depends on the particular experiment. In fact, the Zeeman QI has no projection on the signals detected on the "dipolar channel" (channel $y$ in Eq. (5)) after the JB sequence, which is equivalent to considering $\beta_{Z}=0$. It is worth to mention that $S(t)$ is symmetric with respect to times $\tau$ and $t$. This property, of the JB sequence, demonstrated in the Appendix, will be very important in our following treatment of the observed signals. In the case that the only QI is the total dipolar energy $\mathcal{H}_{D}^{0}$, as is the case of systems with regularly distributed nuclear spins, the dipolar signal of Eq. (5) is proportional to the time derivative of the FID signal. ${ }^{35}$ 


\section{EXPERIMENTAL}

The interest herein is to explore the manifestation of multiple QIs after the Jeener-Broekaert rf pulse sequence. With this aim we analyze the JB signals as a function of the preparation time $\tau$ in different kinds of dipolar networks, with particular attention on long $\tau$.

Experiments at $20 \mathrm{MHz}$ were carried on in a Bruker Minispec mq20 and those at $60 \mathrm{MHz}$ in a homemade pulsed NMR spectrometer based on a Varian EM360 magnet. ${ }^{37,38}$

As shown in Fig. 1, the response of the different compounds to variations of the preparation time is diverse, however they all share the characteristic of being symmetric with respect to $\tau$ and $t$. In powder adamantane (Fig. 1(a)), the signal is proportional to the time derivative of the FID, as shown in Refs. 19 and 38, and keeps this shape for every preparation time $\tau$, which is consistent with the occurrence of only one dipolar constant of motion: the dipolar energy, $\left\langle\mathcal{H}_{D}^{0}\right\rangle .^{4,39}$

On the contrary, in the other compounds shown in Fig. 1 [(b) gypsum and (c) $N$-(4-methoxybenzylidene)-4butylaniline (MBBA)], the dipolar signal shapes change drastically with $\tau$, being proportional to the time derivative of the FID only for short $\tau$ (more precisely, the proportionality holds while $\tau<t_{0}$, where $t_{0}$ is the time at which the time derivative of the FID first crosses through zero). In LCs, a new behaviour of the dipolar signals arises clearly for longer $\tau$ : the signals show an "echo" like aspect, that is, they have a peaked shape whose absolute maximum occurs at a time $t$ from the read pulse equal to the preparation time $\tau$ (see Fig. 1(c)). Such behaviour is not observed in gypsum. (a)
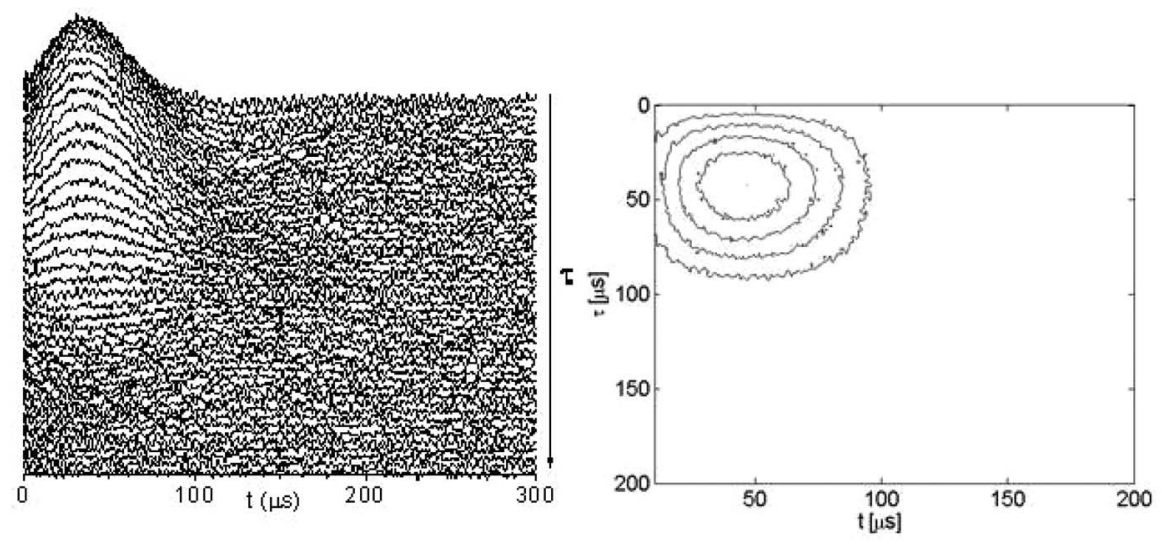

(b)

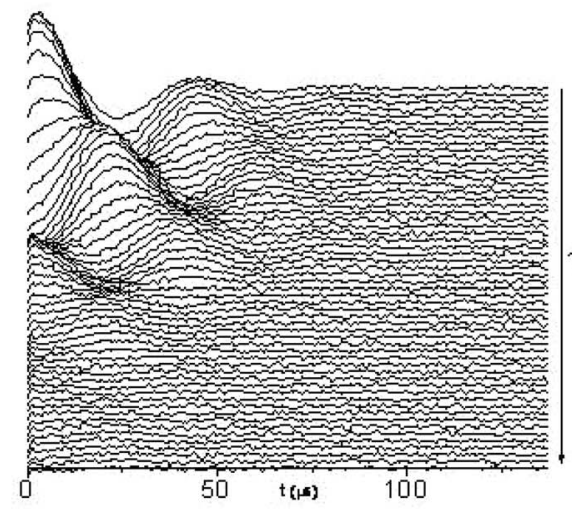

(c)

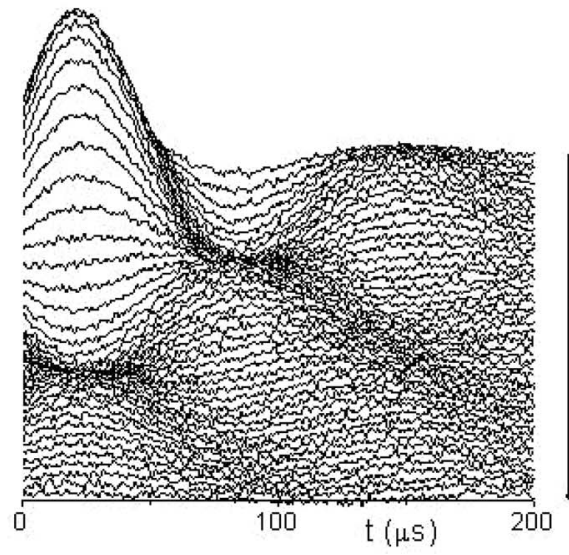

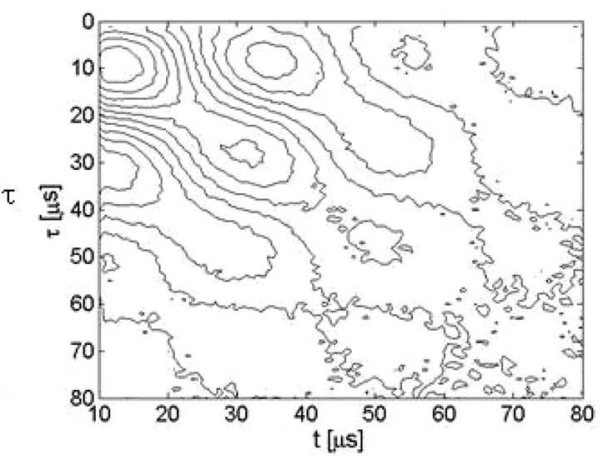

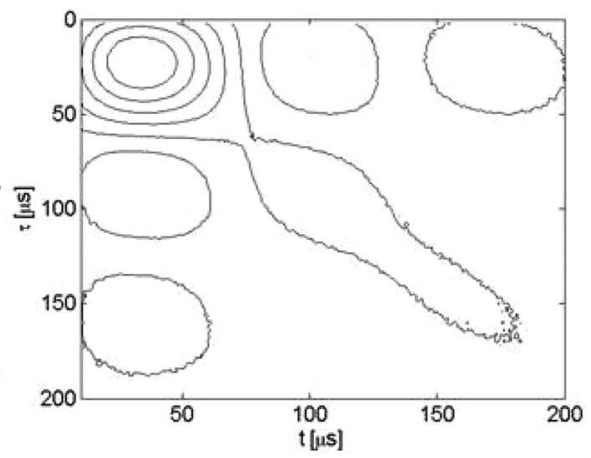

FIG. 1. Stackplots (left column) and contour plots (right column) of the JB signals as a function of the preparation time $\tau$ in (a) adamantane, (b) gypsum, and (c) MBBA liquid crystal. In the stackplots $\tau$ grows downwards within the same range than $t$. 

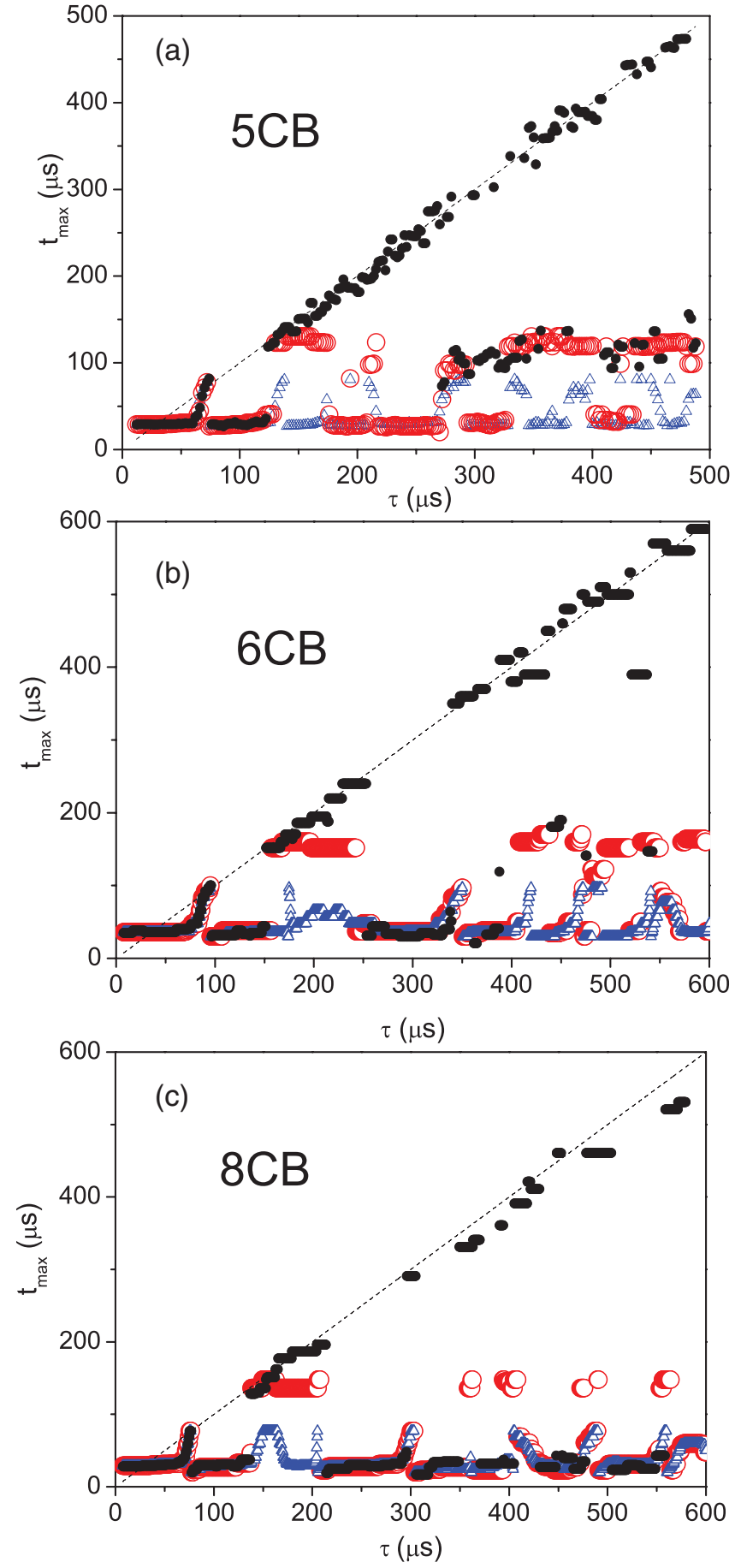

FIG. 2. Acquisition time $t_{\max }$ at which the signals attain their maximum amplitude as a function of the preparation time $\tau$ in the nematic phase of $5 \mathrm{CB}$ (top), 6CB (middle), and 8CB (bottom). Solid circles represent the experimental data. Open triangles and open circles correspond to $t_{\max }(\tau)$ simulated from Eq. (5) with two and three quasiinvariants, respectively. QIs selected as indicated in Table I.

The contour plots on the right column of Fig. 1 also display this characteristic: there is only one peak in adamantane; maxima and minima regularly alternate their positions within the whole $t-\tau$ range in gypsum and, contrastingly, the absolute maxima of the MBBA signals concentrate along the $t=\tau$ diagonal. In order to highlight this feature, Figs. 2 and 3 show the time $t_{\max }$ at which the dipolar signal attains its abso-
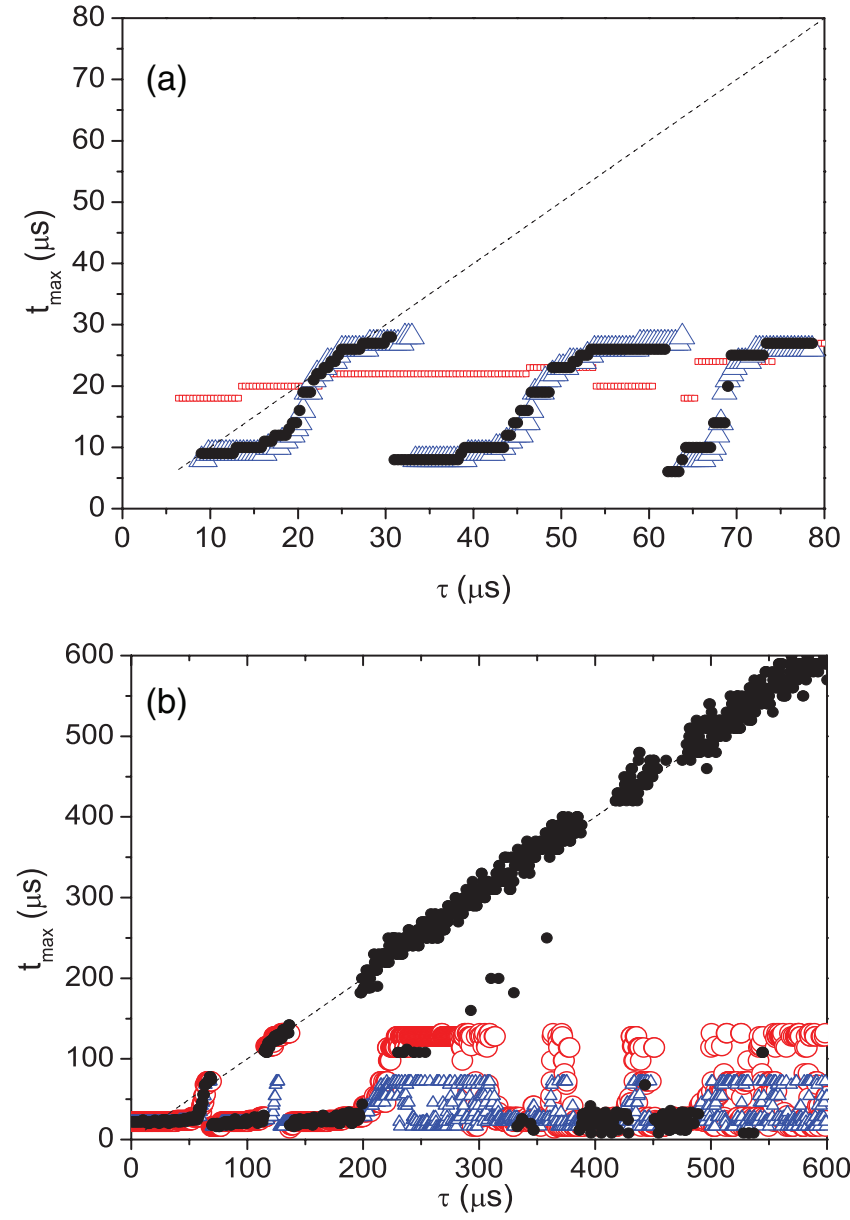

FIG. 3. Acquisition time $t_{\max }$ at which the signals attain their maximum amplitude as a function of the preparation time $\tau$ in a gypsum single crystal (top) and in nematic MBBA (bottom). Solid circles represent the experimental data. Open triangles and open circles correspond to $t_{\max }(\tau)$ simulated from Eq. (5) with two and three quasiinvariants, respectively. QIs selected as indicated in Table I. Solid circles on the gypsum graph correspond an orientation $\vec{B} \|[010]$, while open squares to an orientation that makes the protons at the water molecules equivalent.

lute maximum within the detection period, as a function of the preparation time $\tau$, in different compounds. Preparation times were varied within the range where the $\mathrm{S} / \mathrm{N}$ ratio is greater than $1 \%$ on each compound. It can be seen that the experimental $t_{\max }(\tau)$ (solid circles in Fig. 2) is very similar in all the cyanobiphenyl samples: $4^{\prime}$-n-4-biphenyl-carbonitrile, where $n$ stands for pentyl (5CB), hexyl (6CB), and octyl (8CB). Data from nematic MBBA at the bottom of Fig. 3 behave just as the other nematic LC samples. This behaviour is drastically different in the gypsum single crystal (top of Fig. 3) since $t_{\max }(\tau)$ is bounded in gypsum while grows linearly for long $\tau$ in all the LCs. As seen in this figure, the whole observable range on gypsum is rather smaller than that of the LCs, and the remarkable difference is that LCs develop the echo-like behaviour at preparation times near one fourth of their whole observable ranges while gypsum never does. It is also worth to note that the behaviour of $t_{\max }(\tau)$ is also different in two orientations of the gypsum single crystal with respect to the external magnetic field. The wavy curve of solid circles in Fig. 3(top) corresponds to the orientation $\vec{B} \|[010]$ while the 
open squares correspond to an orientation that makes the protons at the water molecules equivalent.

\section{A. Isolation of the quasi-invariants}

It has been already shown that two independent QIs $(\mathcal{S}$ and $\mathcal{W}$ ) can be prepared in nematic LCs and some hydrated salts, ${ }^{14,36}$ with the property that $\mathcal{W}$ emerges when the weight of $\mathcal{S}$ becomes zero. In fact, the set of dipolar signals in 5CB prepared with $\tau<80 \mu$ s were satisfactorily reproduced by using such two QIs in Eq. (5). ${ }^{14}$ However, this procedure does not describe the signal behaviour for $\tau>80 \mu \mathrm{s}$. Now, we propose a procedure to extract the QIs from the experimental data, under the sole assumption that the states prepared in the experiment are described by Eq. (4). It might be expected that new QIs, if observable, will arise sequentially as the preparation time increases and that in a favourable condition, a subset of preparation times $\left\{\tau_{i}\right\}$ exists at which one QI (the $i$ th) prevails over the others. This amounts to proposing that it is experimentally possible to isolate a subset of QIs. The validity of such working hypothesis will be tested along this work.

The measured data sets are composed by the 2D time domain signals obtained for a set $\left\{\tau_{m}\right\}$ of preparation time values . Let us call $\mathcal{M}\left(\tau_{m}, t\right)$ to the acquired signals after the JB sequence, which we arrange as the rows of the data array, and call $\mathcal{M}\left(\tau, t_{i}\right)$ to the columns of such array (pseudo-signals) which corresponds to a cut of the 2D array at fixed $t=t_{i}$. It is worth to mention that $\mathcal{M}(\tau, t)$ is strictly symmetric in $t$ and $\tau$ in the whole observable time window.

With the aim of deriving the functions $\mathcal{F}_{i}(t)$ from the experimental data we followed these steps:

- Recognize the preparation time $\tau=\tau_{1}$ which makes $\mathcal{M}\left(\tau_{1}, t\right)$ proportional to the time derivative of the FID signal and has maximum amplitude. This signal attains its absolute maximum at $t=t_{1}=\tau_{1}$. Based on former evidence that the state prepared with a time $\tau=\tau_{1}$ is a QI, we assume that the experimental function $\mathcal{M}\left(\tau_{1}, t\right)$ can be identified with the contribution of the first QI to the signal, $\mathcal{F}_{1}(t)$ (see dotted line in Fig. 4).

- Select the experimental pseudo-signal $\mathcal{M}\left(\tau, t_{1}\right)$ and identify it with the "weight" $\beta_{1}(\tau)$ of the first QI.

- Generate the one-QI dipolar signal (as a function of $t$ and $\tau$ ),

$$
\mathrm{S}_{1}(\tau, t)=\beta_{1}(\tau) \mathcal{F}_{1}(t)
$$

In the case of adamantane, the only dipolar QI is the dipolar energy, the 2D signals of Fig. 1(a) can be adequately reproduced with $\mathrm{S}_{1}(\tau, t)$ from Eq. (6). However, this function does not describe the experimental signals of the other studied samples, then, it is necessary to select the contribution of the next QI, $\mathcal{F}_{2}(t)$.

- Analyze the difference between the measured data and the contribution of the first QI to the signal

$$
\mathcal{C}_{2}(\tau, t)=\mathcal{M}(\tau, t)-\mathrm{S}_{1}(\tau, t) .
$$

This data set attains its absolute maximum at a time which we call $t_{2}=\tau_{2}$. Then choose $\mathcal{F}_{2}(t) \equiv \mathcal{C}_{2}\left(\tau_{2}, t\right)$

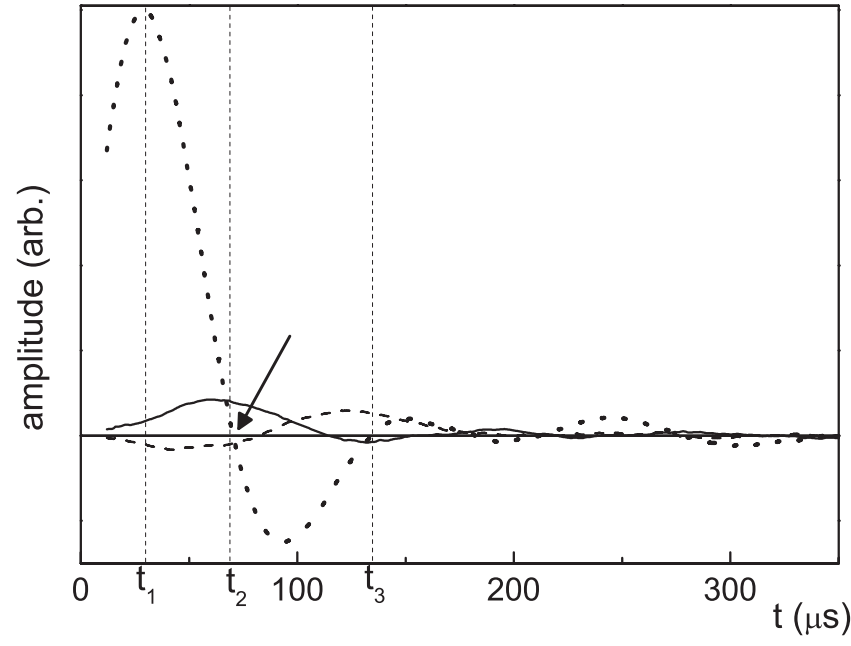

FIG. 4. Signals $\mathcal{F}_{1}$ (dotted), $\mathcal{F}_{2}$ (solid), and $\mathcal{F}_{3}$ (dashed) for $5 \mathrm{CB}$ at $302 \mathrm{~K}$. Vertical lines indicate the times $t_{1}, t_{2}$, and $t_{3}$ of the maximum amplitudes of the consecutive residues. The arrow points to the time at which the first QI has negligible contribution.

and $\beta_{2}(\tau) \equiv \mathcal{C}_{2}\left(\tau, t_{2}\right)$, the solid curve in Fig. 4 . It is worth to notice that $\beta_{1}\left(\tau_{2}\right)=0$, since $\tau_{2}$ is the time at which the first dipolar QI has negligible contribution to the observed signal, as indicated with an arrow in Fig. 4. (This feature agrees with previous works. ${ }^{14,19,36}$ )

- Generate the two-QI dipolar signal

$$
\mathrm{S}_{2}(\tau, t)=\beta_{1}(\tau) \mathcal{F}_{1}(t)+\beta_{2}(\tau) \mathcal{F}_{2}(t) .
$$

This election of $\mathcal{F}_{1}$ and $\mathcal{F}_{2}$ for calculating the NMR signal $\mathrm{S}_{2}(\tau, t)$ yields the curves in open triangles in Figs. 2 and 3 (blue triangles). It allows for an excellent description of the experimental curve in gypsum. In fact, as seen in the top of Fig. 3 both curves (solid circles and open triangles) coincide in almost all the timescale. The dipolar signal shape had already been accounted for within a restricted interval of preparation times by Dumont et al..$^{36}$ by assuming the occurrence of two QIs. Now we learn that these two QIs can give a good description within an extended timescale.

On the contrary, in LC samples $(5 \mathrm{CB}, 6 \mathrm{CB}, 8 \mathrm{CB}$, and MBBA) the triangle-curves only reproduce the experimental behaviour of $t_{\max }(\tau)$ for times $\tau, t<120 \mu \mathrm{s}$, that is, when the preparation times are confined roughly to within the first half period of the dipolar coupling (and exceptionally within small windows at longer $\tau$ values). This failure in describing $t_{\max }(\tau)$ for longer times with only two QIs led us to continue with the procedure in order to find a new curve $\mathcal{F}_{3}$. Again we analyze the residue

$$
\mathcal{C}_{3}(\tau, t)=\mathcal{M}(\tau, t)-\mathrm{S}_{2}(\tau, t)
$$

to find the time $t_{3}=\tau_{3}$ at which this data set attains its absolute maximum and define $\mathcal{F}_{3}(t) \equiv \mathcal{C}_{3}\left(\tau_{3}, t\right)$ (see dashed curve in Fig. 4) and $\beta_{3}(\tau) \equiv \mathcal{C}_{3}\left(\tau, t_{3}\right)$. The times $t_{1}, t_{2}$, and $t_{3}$ corresponding to the different compounds are summarized in Table I.

This selection allows calculating the three-QI signal

$$
\mathrm{S}_{3}(\tau, t)=\beta_{1}(\tau) \mathcal{F}_{1}(t)+\beta_{2}(\tau) \mathcal{F}_{2}(t)+\beta_{3}(\tau) \mathcal{F}_{3}(t),
$$


TABLE I. Preparation/observation times $t_{1}, t_{2}$, and $t_{3}$ found during the procedure used for isolating the first three QIs for each compound and the corresponding temperature. Notice that $t_{i}$ values in 5CB change with temperature.

\begin{tabular}{lcccc}
\hline \hline Compound & Temperature $(\mathrm{K})$ & $t_{1}(\mu \mathrm{s})$ & $t_{2}(\mu \mathrm{s})$ & $t_{3}(\mu \mathrm{s})$ \\
\hline $5 \mathrm{CB}$ & 302 & 30 & 70 & 134 \\
$5 \mathrm{CB}$ & 297 & 26 & 60 & 106 \\
$6 \mathrm{CB}$ & 297 & 36 & 85 & 160 \\
$8 \mathrm{CB}$ & 311 & 29 & 74 & 140 \\
Gypsum & 311 & 10 & 28 & $\ldots$ \\
MBBA & 311 & 22 & 65 & 120 \\
\hline \hline
\end{tabular}

which improves the calculated curve for $t_{\max }(\tau)$. Figures 2(a)2(c) and 3(b) show that involving a third QI yields the open (red) circles curve which gives a noticeably better description of the experimental curves. Particularly, they coincide even within $120 \mu \mathrm{s}<\tau<150 \mu$ s where the echo-like behaviour starts. Figure 5 shows a detail of $t_{\max }(\tau)$ in MBBA, where the three-QI curve is definitely better than the two-QI curve especially within the interval $115 \mu \mathrm{s}<\tau<136 \mu \mathrm{s}$, where the third QI becomes dominant. However, it still does not account for the echo-like behaviour for longer preparation times. Low signal-to-noise ratio did not allow us to continue with the procedure to obtain further QIs.

\section{B. Relaxation times}

In order to test if $\mathcal{F}_{3}(t)$ does in fact behave as the signal of a QI, we analyze the signal amplitude attenuation as a function of the evolution time $t_{e}$ as in a regular spin-lattice relaxation experiment. Figure 6 shows amplitude attenuation of $\mathcal{F}_{1}(t), \mathcal{F}_{2}(t)$, and $\mathcal{F}_{3}(t)$ in $5 \mathrm{CB}$ at $302 \mathrm{~K}$, for the preparation times shown in Table I: $\tau=30 \mu \mathrm{s}$, circles; $\tau=70 \mu \mathrm{s}$, open squares; and $\tau=134 \mu \mathrm{s}$, full squares. The three relaxation curves can be adequately fitted with two exponential decays, the common short time decay (the same for all) is $4 \mathrm{~ms}$ while the longer characteristic times are $T_{D 1}=67 \mathrm{~ms}$ (circles), $T_{D 2}$ $=56 \mathrm{~ms}$ (open squares), and $T_{D 3}=39 \mathrm{~ms}$ (full squares). The

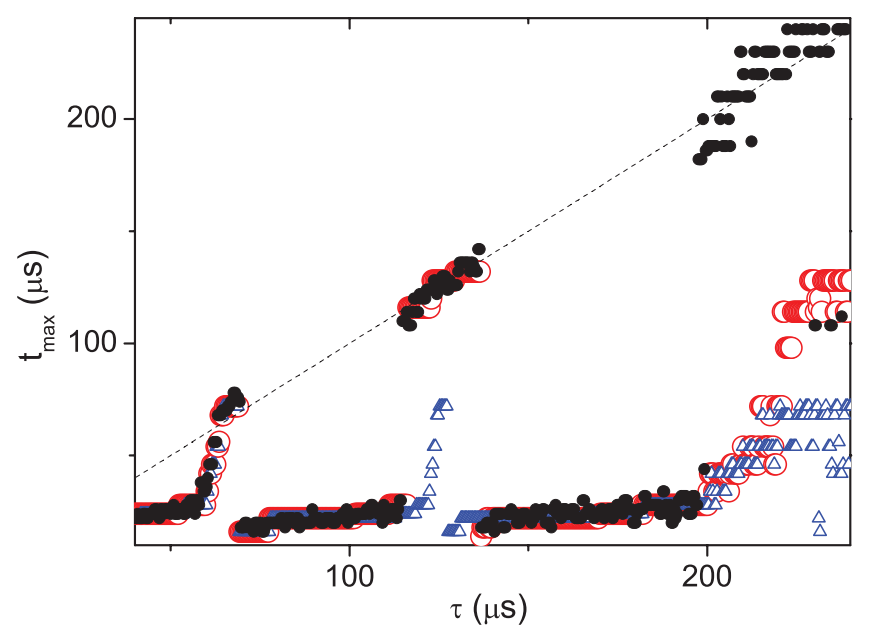

FIG. 5. Detail of the experimental $t_{\max }(\tau)$ in nematic MBBA (solid circles) and its description with Eq. (5) considering two QI (open triangles) and three QI (open circles).

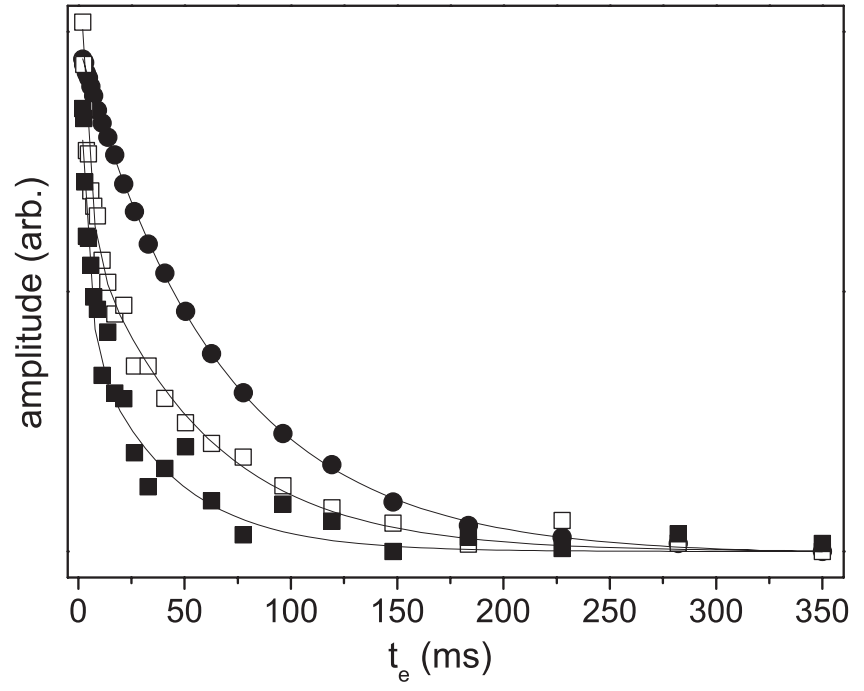

FIG. 6. Spin-lattice relaxation curves of three QIs on 5CB at $302 \mathrm{~K}$ prepared with different preparation times: $\tau=30 \mu \mathrm{s}$, circles; $\tau=70 \mu \mathrm{s}$, open squares; and $\tau=134 \mu$ s, full squares.

fast decay is just a witness of the attenuation of higher order coherences, in fact, its characteristic time coincides with the timescale of irreversible decoherence as measured in Ref. 3. On the other hand, the longer decay times are all of the order of the dipolar relaxation time (67 $\mathrm{ms}$ in this case).

\section{Multiple quantum coherence contents}

In Ref. 19 it was shown that the QE state which is proportional to $\mathcal{H}_{\mathcal{S}}$ in $5 \mathrm{CB}$ (prepared by setting $\beta_{\mathcal{W}}\left(\tau_{1}\right) \simeq 0$ ) is a two-spin correlated state since it involves at most two-spin tensors, while states proportional to $\mathcal{H}_{\mathcal{W}}$ (prepared by setting $\beta_{\mathcal{S}}\left(\tau_{1}\right) \simeq 0$ ) involve correlations of more-than-two spin tensors. With the aim of studying the correlated nature of the states prepared at different $\tau$ 's in 5CB, we analyze the multiple quantum coherence content on the $\mathrm{X}$-basis using the same pulse sequence as in Refs. 10 and 19. In this experiment, rotating the state around an axis orthogonal to $\mathrm{Z}$ allows encoding MQC which reflect the number of multiply connected spins $1 / 2$ in the prepared states. The experiment begins by preparing the state with the phase shifted JB pulse pair, then, a waiting time $t_{w}$ is followed by two pulses $90_{(\phi+\pi / 2)}-\epsilon-90_{(y)}$ which encode the coherence numbers of the quantum state at time $t_{w}$ in the $\mathrm{X}$-basis, when varying $\phi$ systematically in successive experiments. Fourier transformation of the signal amplitude with respect to $\phi$ yields an X-basis coherence spectrum. Our experiment, conducted on 5CB at $297 \mathrm{~K}$ and 60 $\mathrm{MHz}$, was set to encode up to 8-quantum coherences on the $\mathrm{X}$-basis, however, coherences higher than 4 in 5CB were seen to fall below the noise level. The MQC content varies with the preparation time $\tau$, as shown in Fig. 7(a). As expected, zero and double quantum coherences are dominant for $0<\tau$ $<50 \mu \mathrm{s}$. The fact that the quotient of their amplitudes is ca. 1.5 indicates that the states prepared within this interval can be described with only one QI (or dipolar constant of motion), ${ }^{10}$ which is proportional to $\mathbf{T}_{20}$, the 0 component of 


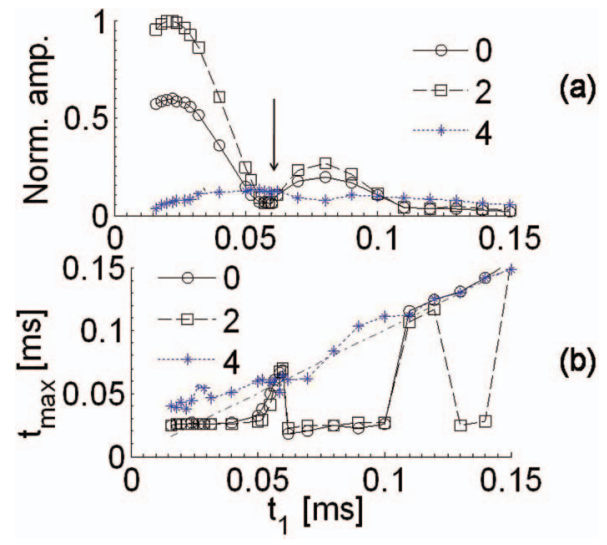

FIG. 7. (a) Maximum amplitude attained by each coherence, normalized to coherence 2; (b) observation-time window at which the maximum amplitudes occur.

a spherical tensor of rank 2. Coherences \pm 4 instead, grow slowly with $\tau$ and dominate only within restricted intervals.

At a preparation time near $60 \mu$ s the ratio between coherences 2 and 0 changes drastically and their amplitudes become comparable to the rising 4-quantum coherence (see vertical arrow in Fig. 7(a)). As shown in the second row of Table I, this is precisely the preparation time at which $\mathcal{F}_{2}(t)$, the signal associated with the second QI, attains its maximum, showing that these states involve more-than-two spin correlations (or multi-spin-correlated states). Notice that also near $110 \mu \mathrm{s}$ all the coherences attain comparable amplitudes, and this preparation time coincides with the third QI (see Table I).

We observed that the relative amplitudes of the various MQC components depend on which window along the acquisition time is used for calculating the MQC spectrum. This feature is shown in Fig. 7(b) where the time interval (along the acquisition period) at which the absolute maximum value of each coherence component occurs is plotted as a function of the preparation time. The salient feature is that different coherences have a very different behaviour. The largest contribution to coherences 0 and \pm 2 on the X-basis come from the window near $t_{\max }=30 \mu \mathrm{s}$, except within the narrow preparation intervals which correspond to the second and third QIs. On the contrary, the largest amplitude of coherences \pm 4 (on the X-basis) falls on the line $t_{\max }=\tau$, in other words, it behaves like an echo. This distinct behaviour agrees with a view that multi-spin correlations arise at longer times than two-spin correlations, and also that the echo like signals prepared with $\tau>70 \mu$ s correspond to states of multi-spin nature.

\section{DISCUSSION AND CONCLUSIONS}

In this work we present an experimental survey on the occurrence of several QIs created by means of the JB sequence, in different dipolar-coupled spin systems. To account for the dependence of the JB signals on the preparation time, we propose a generalization of the method presented in Refs. 14 and 36. Starting from the hypothesis that the quasi equilibrium states can be written as a linear combination of a complete set of QIs, that is, operators which commute with $\mathcal{H}_{D}^{0}$, we demonstrate that, in fact, multiple QIs can be prepared in the dipole interacting proton spin system on LC molecules, in consistency with the expectation that $\mathrm{N}$ interacting-spin clusters should in principle allow the occurrence of many constants of motion.

The well known dipolar-order state, which is a two-spin object, comes at short preparation times, while the other observed QIs emerge sequentially at longer preparation times. We propose a criterion to experimentally isolate each QI and we observe that the first three QIs in 5CB have evidently distinct spin-lattice relaxation times, as expected for true quasiinvariants.

Though at present the tensor structure of these QIs is still unknown, the multiple quantum coherence experiments demonstrate that they have multi-spin character, which implies that their tensor structure must involve products of individual angular momentum operators of many spins. Also, we verified that the observed echo-like behaviour of the dipolar signal in LC for long preparation times is also a consequence of this characteristic.

In this way we demonstrate that the initial Zeeman order (at thermal equilibrium) can in fact be transferred to three dipolar constants of motion in LC within the scanned short and intermediate $\tau$ ranges. On the contrary, only two constants of motion were observed in gypsum when the magnetic field $\vec{B} \|[010]$ and only one at an orientation that makes the protons at the water molecules equivalent. The fact that compounds having very different numbers of interacting spins (11-25 in LCs and infinite in gypsum) admit the preparation of more than one dipolar QI, indicates that their occurrence is not exclusively determined by the size of the dipolar network. Instead, both the occurrence of multiple QIs and the many-spin character they show, do depend strongly on the topology of the spin network, since it determines the quantum dynamics of the spin system. This assertion also agrees with the fact that the range whereof our three-QI model description provides a suitable description varies from one LC to another. Accordingly, one might expect that considering more QIs would extend the description to longer preparation times.

The experimental evidence of multiple QIs presented here seems to dissent with the statement that many-spin constants of motion could only be expectable in very small (less than 9) dipole coupled spin systems. ${ }^{2}$ However, that prediction comes out from the numerical analysis on a linear chain of equidistant spins, so the possible cause of the vanishing of such constants of motion might be attributed to the regularity of the chosen system instead of its size.

It is worth to note that the feasibility of transferring the Zeeman order to multi-spin QE states is not an exclusive feature of spin clusters as the proton system in LCs as illustrated in this work. In fact, the "interpair" state observed in hydrated crystals admits multiple quantum coherences up to the fourth order on the $X$-basis, implying four-spin correlations. ${ }^{19}$ Then the question arises about which are the necessary characteristics the dipolar network must have in order to admit the preparation of more-than-one dipolar QI. A coarse criterion could be (i) the occurrence of a doublet in the NMR spectrum, which generally implies the possibility of classifying the dipolar couplings according to their intensity into "strong" and "weak", and (ii) the possibility of truncating the weaker 
term of the dipolar Hamiltonian with respect to the stronger term. According to Ref. 40, in order to be able to separate a strong interaction, as could be the nearest dipole pair (giving a coherent response), a particular relation between the dipolar coupling of the pair and the total sum of the squared couplings of the crystal must be satisfied. For example, in $\mathrm{CaF}_{2}$ the structure of the dipolar network is almost in the threshold of resolving the doublet in the [100] direction. The case of $5 \mathrm{CB}$ and other LC molecules is very different, with the consequent well resolved doublet.

According to our results, in LCs the two-spin correlations dominate the coherent multi-spin dynamics during the early timescale of the preparation period. Within such range of $\tau$ values, however, there are narrow time windows where these correlations vanish and higher order correlations can efficiently give place to multi-spin order QE states. Finally, for longer $\tau$ values the two-spin correlations would turn inefficient to generate two-spin order, and the multi-spin correlations would be responsible for the echo-like behaviour of the NMR signal. The behaviour of the signal for long preparation times in gypsum is rather different. In this case, the echolike shift of the signal is not observed, which suggests that, although the lattice may admit the generation of multi-spin correlations, the two-spin correlations generated during the preparation period seem to influence the creation of multispin QIs even for long preparation times.

In summary, we found experimental evidence of existence of multiple quasi-invariants in liquid crystals NMR that can be created with the JB sequence, and explained the observed echo-like behaviour as being consequence of the generation of many quasi-equilibrium states as the preparation time of the JB experiment gets longer.

We interpreted the response of the spins for long times in the Jeener-Broekaert experiment as a hallmark of the multispin dynamics which develops during the preparation period, which strongly depends on the topology of the dipolar network. These ideas were probed by means of meticulous experiments on a variety of LC and solid samples. Particularly, we associated the echo-like behaviour, ubiquitously found in LCs, with the multispin nature of the new detected quasiinvariants.

\section{ACKNOWLEDGMENTS}

We thank J. Jeener for inspiring discussions which helped us to perceive the importance of the problem of multiple quasi invariants in liquid crystals. This work was supported by SECYT Universidad Nacional de Córdoba and MINCYT Córdoba. C.J.B. and H.H.S. thank Consejo Nacional de Investigaciones Científicas y Técnicas, CONICET for financial support.

\section{APPENDIX: SYMMETRIC CHARACTER OF THE DIPOLAR SIGNAL}

The interest here is to show that the set of NMR signals of Eq. (5) is symmetric in the preparation and observation times. To our knowledge, this property has not been given a theoretical treatment. We first write the density operator after the third pulse of the Jeener-Broekart sequence and then calculate the NMR signal. Let us represent the radiofrequency pulse of phase $\alpha$ and angle $\theta$ by the operator

$$
P_{\alpha}(\theta)=e^{-i \alpha I_{z}} e^{i \theta I_{x}} e^{i \alpha I_{z}},
$$

where $\alpha=0$ corresponds to a pulse in phase with the rotating frame (or $x$ direction) and $\alpha=90^{\circ}$ to a pulse $90^{\circ}$ out of phase with respect to the rotating frame (or $y$ direction). This expression for $P_{\alpha}(\theta)$ corresponds to on-resonance, hard pulses as used in our experiment. The free evolution operator is

$$
U(s)=\exp \left\{-i \mathcal{H}_{D}^{0} s\right\},
$$

where $s$ stands for the preparation period $\tau$, evolution after the second pulse $t_{e}$, or the observation time $t$.

The experiment starts from thermal equilibrium

$$
\rho_{0}=\frac{e^{-\beta_{0} \mathcal{H}_{S}}}{\mathcal{Z}}
$$

where $\beta_{0}$ is the lattice inverse temperature and $\mathcal{H}_{S}$ as in Eq. (2) and $\mathcal{Z}$ is the normalization constant. In the high field, high temperature approximation

$$
\rho_{0} \sim\left(\mathbf{1}-\beta_{0} \mathcal{H}_{Z}\right) / \mathcal{Z}=\left(\mathbf{1}+\beta_{0} \omega_{0} I_{Z}\right) / \mathcal{Z}
$$

Then, the action of the first pulse is $P_{x}\left(\frac{\pi}{2}\right) \rho_{0} P_{x}^{\dagger}\left(\frac{\pi}{2}\right)=-I_{y}$, and the state at time $t$ after the third pulse can be written as

$$
\begin{aligned}
\rho\left(\tau, t_{e}, t\right)= & \frac{1}{\mathcal{Z}} U(t) P_{y}\left(\frac{\pi}{4}\right) U\left(t_{e}\right) P_{y}\left(\frac{\pi}{4}\right) U(\tau)\left(\mathbf{1}-\beta_{0} \omega_{0} I_{y}\right) \\
& \times U^{\dagger}(\tau) P_{y}^{\dagger}\left(\frac{\pi}{4}\right) U^{\dagger}\left(t_{e}\right) P_{y}^{\dagger}\left(\frac{\pi}{4}\right) U^{\dagger}(t),
\end{aligned}
$$

where

$$
P_{\alpha}^{\dagger}(\theta)=e^{i \alpha I_{z}} e^{-i \theta I_{x}} e^{-i \alpha I_{z}}=P_{\alpha}^{*}(\theta)
$$

and

$$
U^{\dagger}(s)=\exp \left\{i \mathcal{H}_{D}^{0} s\right\}=U^{*}(s) .
$$

Let us divide the evolution after the second pulse as $U\left(t_{e}\right)$ $=U\left(\frac{t_{e}}{2}\right) U\left(\frac{t_{e}}{2}\right)$ and define

$$
L(s)=U\left(\frac{t_{e}}{2}\right) P_{y}\left(\frac{\pi}{4}\right) U(s) .
$$

Using Eqs. (A3) and (A4) we see that

$$
\begin{gathered}
L^{*}(s)=U^{\dagger}\left(\frac{t_{e}}{2}\right) P_{y}^{\dagger}\left(\frac{\pi}{4}\right) U^{\dagger}(s), \\
L^{\dagger}(s)=U^{\dagger}(s) P_{y}^{\dagger}\left(\frac{\pi}{4}\right) U^{\dagger}\left(\frac{t_{e}}{2}\right), \\
L^{\dagger *}(s)=U(s) P_{y}\left(\frac{\pi}{4}\right) U\left(\frac{t_{e}}{2}\right) .
\end{gathered}
$$

Then, the signal after the third pulse is $S\left(\tau, t_{e}, t\right)=\operatorname{Tr}$ $\left\{I_{y} \rho\left(\tau, t_{e}, t\right)\right\}$, and using that $\operatorname{Tr}\left\{I_{y}\right\}=0, I_{y}=-I_{y}^{*}$, and the properties from Eqs. (A5) leaves

$$
\begin{aligned}
S\left(\tau, t_{e}, t\right) & =\frac{-\beta_{0} \omega_{0}}{\mathcal{Z}} \operatorname{Tr}\left\{I_{y} L^{\dagger *}(t) L(\tau) I_{y} L^{\dagger}(\tau) L^{*}(t)\right\} \\
& =\frac{\beta_{0} \omega_{0}}{\mathcal{Z}} \operatorname{Tr}\left\{\left[L(t) I_{y} L^{\dagger}(t)\right]^{*} L(\tau) I_{y} L^{\dagger}(\tau)\right\}
\end{aligned}
$$

Now, recalling that the signal is real,

$$
\begin{aligned}
S\left(\tau, t_{e}, t\right) & =S^{*}\left(\tau, t_{e}, t\right) \\
& =\frac{\beta_{0} \omega_{0}}{\mathcal{Z}} \operatorname{Tr}\left\{\left[L(\tau) I_{y} L^{\dagger}(\tau)\right]^{*} L(t) I_{y} L^{\dagger}(t)\right\} .
\end{aligned}
$$


Then

$$
S\left(\tau, t_{e}, t\right)=S\left(t, t_{e}, \tau\right)
$$

where the roles of $t$ and $\tau$ are interchanged, which shows that $S\left(\tau, t_{e}, t\right)$ is symmetric in $t$ and $\tau$ with independence of the value of $t_{e}$.

${ }^{1}$ H. H. Segnorile and R. C. Zamar, J. Chem. Phys. 135, 244509 (2011).

${ }^{2}$ J. D. Walls and Y. Lin, Solid State Nucl. Magn. Reson. 29, 22 (2006).

${ }^{3}$ C. E. González, H. H. Segnorile, and R. C. Zamar, Phys. Rev. E 83, 011705 (2011).

${ }^{4}$ J. Jeener and P. Broekaert, Phys. Rev. 157, 232 (1967).

${ }^{5}$ H. Eisendrath, W. Stone, and J. Jeener, Phys. Rev. B 17, 47 (1978).

${ }^{6}$ J. D. van Beek, A. Hemmi, M. Ernst, and B. H. Meier, J. Chem. Phys. 135, 154507 (2011).

${ }^{7}$ T. Charpentier, F. S. Dzheparov, J. F. Jacquinot, and J. Virlet, Chem. Phys. Lett. 352, 447 (2002).

${ }^{8}$ S. I. Doronin, E. Feldman, E. Kuznetsova, G. B. Furman, and S. D. Goren, Phys. Rev. B 76, 144405 (2007); JETP Lett. 86, 24 (2007).

${ }^{9}$ M. E. Halse, J. N. Dumez, and L. Emsley, J. Chem. Phys. 136, 224511 (2012).

${ }^{10}$ H. Cho, D. Cory, and C. Ramanathan, J. Chem. Phys. 118(8), 3686 (2003).

${ }^{11}$ J. Baum, M. Munowitz, A. N. Garroway, and A. Pines, J. Chem. Phys. 83, 2015 (1985)

${ }^{12}$ A. Keller, Adv. Magn. Reson. 12, 183 (1988).

${ }^{13}$ H. Schmiedel, S. Grande, and B. Hillner, Phys. Lett. 91A, 365 (1982).

${ }^{14}$ O. Mensio, C. E. González, and R. C. Zamar, Phys. Rev. E 71, 011704 (2005).

${ }^{15}$ H. H. Segnorile, C. J. Bonin, C. E. González, R. H. Acosta, and R. C. Zamar, Solid State Nucl. Magn. Reson. 36, 77 (2009).

${ }^{16}$ D. P. Weitekamp, Adv. Magn. Reson. 11, 111 (1983).

${ }^{17}$ R. G. C. Mc. Elroy, R. T. Thompson, and M. M. Pintar, Phys. Rev. A 10, 403 (1974).

${ }^{18}$ F. Noack, St. Becker, and J. Struppe, Annu. Rep. NMR Spectrosc. 33, 1-36 (1996).

${ }^{19}$ L. Buljubasich, G. A. Monti, R. H. Acosta, C. J. Bonin, C. E. González, and R. C. Zamar, J. Chem. Phys. 130, 024501 (2009).

${ }^{20}$ R. Zamar, E. Anoardo, O. Mensio, D. Pusiol, S. Becker, and F. Noack, J. Chem. Phys. 109(3), 1120 (1998).
${ }^{21}$ O, Mensio, R. Zamar, E. Anoardo, R. H. Acosta, and R. Y. Dong, J. Chem. Phys. 123, 204911 (2005).

${ }^{22}$ S. Emid, J. Smidt, and A. Pines, Chem. Phys. Lett. 73, 496 (1980).

${ }^{23}$ B. Furman and S. D. Goren, J. Phys.: Condens. Matter 17, 4501 (2005).

${ }^{24}$ S. Matsui, S. Saito, T. Hashimoto, and T. Inouye, J. Magn. Reson. 160, 13 (2003).

${ }^{25}$ T. S. Mahesh and D. Suter, Phys. Rev. A 74, 062312 (2006).

${ }^{26}$ H. G. Krojanski and D. Suter, Phys. Rev. Lett. 93, 090501 (2004); 97, 150503 (2006).

${ }^{27}$ D. A. Lidar, I. L. Chuang, and K. B. Whaley, Phys. Rev. Lett. 81, 2594 (1998).

${ }^{28}$ A. K. Khitrin, V. L. Ermakov, and B. M. Fung, Chem. Phys. Lett. 360, 161 (2002).

${ }^{29}$ M. A. Nielsen and I. L. Chuang, Quantum Computation and Quantum Information (Cambridge University Press, Cambridge, 2001).

${ }^{30}$ D. Suter and T. S. Mahesh, J. Chem. Phys. 128, 052206 (2008).

${ }^{31}$ V. I. Yukalov, Phys. Lett. A 376, 550 (2012).

${ }^{32}$ A. Polkovnikov, K. Sengupta, A. Silva, and M. Vengalatore, Rev. Mod. Phys. 83, 863 (2011).

${ }^{33}$ Malcolm H. Levitt, Spin Dynamics, Basics of Nuclear Magnetic Resonance (John Wiley \& Sons, Chichester, 2005).

${ }^{34}$ A. Abragam, The Principles of Nuclear Magnetism (Oxford University Press, London, 1961).

${ }^{35}$ A. Abragam and M. Goldman, Nuclear Magnetism: Order and Disorder, International Series of Monographs in Physics (Clarendon, Oxford, 1982).

${ }^{36}$ E. Dumont, J. Jeener, and P. Broekaert, Phys. Rev. B 49, 6763 (1994).

${ }^{37}$ H. H. Segnorile, Ph.D. thesis, Universidad Nacional de Córdoba, 2009, see http://www2.famaf.unc.edu.ar/publicaciones/documents/serie_d/DFis138. pdf

${ }^{38}$ C. J. Bonin, Ph.D. thesis, Universidad Nacional de Córdoba, 2011, see http://www2.famaf.unc.edu.ar/publicaciones/documents/serie_d/DFis147. pdf

${ }^{39}$ H. Cho, T. D. Ladd, J. Baugh, D. G. Cory, and C. Ramanathan, Phys. Rev. B 72, 054427 (2005).

${ }^{40}$ J. Jensen, Phys. Rev. B 52, 9611 (1995).

${ }^{41}$ M. Levitt, D. Suter, and R. R. Ernst, J. Chem. Phys. 84, 4243 (1986).

${ }^{42}$ H. H. Segnorile and R. C. Zamar, "Quantum irreversible decoherence behaviour in open quantum systems with few degrees of freedom: Application to ${ }^{1} \mathrm{H}$ NMR reversion experiments in nematic liquid crystals," J. Chem. Phys. (to be published); e-print arXiv:1305.0973.

${ }^{43}$ D. Mozyrsky and V. Privman, J. Stat. Phys. 91, 787 (1998). 